\title{
CAPÍTULO XXVII
}

\section{SISTEMATIZACIÓN DE EXPERIENCIAS: UNA PROPUESTA DE INVESTIGACIÓN CUALITATIVA EN ENFERMERÍA}

\author{
Edelsy Benjumea Liñan \\ Magister en Educación, docente investigadora Universidad Popular del Cesar, \\ Colombia. Correo electrónico: edelsybenjumea@unicesar.edu.co. https://orcid. \\ org/0000-0001-5581-6232
}

\section{Luz Divia Mejia Reales}

Magister en Educación, docente investigadora Universidad Popular del Cesar, Colombia. Correo electrónico: luzmejia@unicesar.edu.co

Fabio Hernando Cortes Escalante

Postdoctor en Gerencia en las Organizaciones: docente investigador Universidad Popular del Cesar, Colombia. Correo electrónico: fabiohcortes@unicesar.edu.co

\section{Resumen}

El objetivo de este estudio, fue proponer la Sistematización de Experiencias, como una forma de hacer investigación cualitativa en Enfermería. El abordaje del estudio, se hizo desde la investigación proyectiva, la cual, consiste en la elaboración de una propuesta o un plan, para ayudar en una necesidad de tipo práctico; a partir, de un diagnóstico de necesidades. La muestra fue seleccionada por conveniencia; constituida por 35 estudiantes de sexto semestre, de la asignatura Seminario de Investigación y 10 docentes de Enfermería, que pertenecen al equipo Currículo. Se utilizó, la entrevista individual y el grupo focal, como técnicas de investigación. En los resultados, los docentes y estudiantes, reconocieron la importancia de la Sistematización de Experiencias como investigación cualitativa; método de producción de conocimiento, en la profesión de Enfermería; se evidenció, el desconocimiento de la Sistematización de Experiencias, en las prácticas, actividad que permite comprender el entorno y transformar realidades que se viven.

Palabras clave: enfermería, investigación, reflexión, sistematización de experiencias, acción.

www.doi.org/10.47212/tendencias2020vol.xii.28 


\title{
SYSTEMATIZATION OF EXPERIENCES: A PROPOSAL FOR QUALITATIVE RESEARCH IN NURSING
}

\begin{abstract}
The objective of this study was to propose the systematization of experiences as a way of doing qualitative research in nursing. The study was carried out from projective research, which consists of preparing a proposal or plan, to help with a practical need based on a needs assessment. The sample was selected for convenience, consisting of 35 sixth semester students from the research seminar course and 10 nursing teachers who belong to the curriculum team. The individual interview and the focus group were used as research techniques. In the results, the teachers and students recognized the importance of the systematization of experiences as qualitative research, method of knowledge production in the nursing profession, the lack of knowledge of the systematization of experiences in the practical activities that allows understanding the environment and transforming realities that are lived.
\end{abstract}

Keywords: action, nursing, systematization of experiences, reflection, research.

\section{Proyecto de investigación}

Sistematización de Experiencias en el Programa de Enfermería. Una propuesta de investigación cualitativa, 2017.

\section{Introducción}

El presente estudio, tiene como objetivo, desarrollar una propuesta de Investigación Cualitativa en Enfermería; la Sistematización de Experiencias, como estrategia investigativa, dada su importancia, como un proceso de investigación, que admite comprender la naturaleza de las prácticas, desde la perspectiva de las personas que la han vivido. Por consiguiente, el investigador en este proceso, tiene la ocupación de interpretar y construir los significados subjetivos, que la persona atribuye a dicha experiencia.

Triviño y Sanhueza. (2005), describen la siguiente problemática relacionada con la formación investigativa en Enfermería: 
- Desmotivación para el desarrollo de la investigación, la cual dificulta los procesos de estudio y comprensión de los paradigmas en investigación.

- Formación con limitados conocimientos filosóficos, epistemológicos y metodológicos para la investigación del objeto disciplinar (el cuidado).

- Limitación para analizar críticamente los diferentes paradigmas de investigación.

- Insuficiente abordaje desde el diseño curricular, en el proceso enseñanza aprendizaje, de las diferentes visiones metodológicas, para la comprensión de fenómenos como objeto de estudio.

- La investigación en Enfermería, como asignatura independiente, se encuentra desarticulada con las demás asignaturas básicas, de apoyo y profesional (Alarcón y Astudillo, 2007).

Además los docentes cada uno por su lado aplican sus propios criterios, en muchas ocasiones se orienta la enseñanza aprendizaje sin hacer distinción metodológica de la investigación formativa en el pregrado y la formación en investigación propiamente dicha. No es beneficiosa la pedagogía y la didáctica empleada para que los estudiantes encuentren la necesidad y el sentido de la investigación cualitativa en Enfermería. A esto se suma que más del $90 \%$ de los proyectos de investigación se realizan con enfoque cuantitativo.

Hay que hacer notar, que la construcción del conocimiento en Enfermería en su práctica profesional, contiene problemas de investigación sobre la vida, la salud y la enfermedad del ser humano; esto, demanda una búsqueda, de métodos y técnicas apropiadas; con el interés de comprender el accionar de Enfermería, como disciplina y profesión; en la práctica se descubren significados en la acción de cuidar a las personas; sus sentimientos, motivaciones, emociones, percepciones, entre otros; lo cual, 
se considera relevante, para bridar una atención humanizada; lo que lleva a precisar, que para su abordaje, se requieren los distintos enfoques metodológicos, tanto en la academia, como en la asistencia, para de esta manera, dar solución a la problemática, que a diario, sepresenta en profesionales de la salud . (OPS, OMS, 2013).

Este estudio, plantea la Sistematización de Experiencias, como un interesante elemento de reflexión y acción; para orientar, el proceso de producción de conocimientos en Enfermería; con la intención, de encontrar nuevos caminos epistemológicos y metodológicos, que conduzcan a abordar las situaciones particulares de esta disciplina de la ciencia de la salud.

\section{Fundamentación teórica}

La sistematización de experiencias prácticas, es un proceso pedagógico, que permite la formación de sujetos críticos y propositivos, con capacidades para comprender e incidir, en los diferentes periodos de la vida. (Mejía, 2011). Asimismo, el Consejo Internacional de Enfermería (CIE), plantea que la práctica basada en la investigación se constituye en un distintivo de la Enfermería profesional, para generar nuevos desarrollos teóricos, es decir, permitir el avance de la ciencia; "evaluar el ejercicio y los servicios actuales, como problemática social, que aporta evidencia científica en la formación, la práctica y la gestión del cuidado" (Vélez et al., 2014 p.13).

Estudios realizados en América Latina, recomiendan, para el currículo de Enfermería, iniciar con las bases de la investigación cualitativa desde pregrado, lo que permite, comprender los fenómenos que experimentan los pacientes; "la mayoría de las investigaciones, tienen enfoque cuantitativo, positivista; mientras, que los estudios cualitativos, han sido menos utilizados" (Galeano, 2013 p. 18).

Es preciso señalar, que el profesional de Enfermería, es un educador, mediador en los problemas sociales; interviene en la promoción y prevención de los eventos, que se presentan en el individuo, familia y colectivos; por lo tanto, acumula una gran cantidad de experiencia, por lo que su papel de investigador, en el enfoque de cualitativo, es más relevante porque es el rol propio de sus actividades, ya que la investigación acción es intrínseca a su diario accionar. 
En este sentido, Cifuentes (2006), considera la Sistematización de Experiencia, como la interpretación crítica de una o varias experiencias, que al ser ordenadas y reconstruidas, revela y expone la razón de un acontecimiento vivido, las intervenciones, cómo son las relaciones; por qué se han hecho tal o cual modo. De acuerdo con el autor, a los profesionales de Enfermería, se les facilita aplicar esa herramienta, debido al elevado volumen de situaciones, que generan experiencias, en su cotidianidad.

Estas razones permiten explicar, que la Sistematización de Experiencias tiene una función educativa y un potencial formativo, para quienes se involucran en su ejecución. En otras palabras, puede facilitar cambios en los sistemas cognitivos y afectivos de los actores de la experiencia, mediante el dialogo de saberes y la orientación permanente, entre el investigador y el participante.

Esto, se da por la naturaleza de Enfermería y la interdisciplinariedad e integralidad, para brindar el cuidado; empleando otras disciplinas, que favorecen los resultados de la atención al paciente. Los autores Gennaro y Fehder (2011), explican que la Sistematización de Experiencias, es trascendental, porqueayuda a la ciencia de la Enfermería, permitiendo la producción de conocimientos y teorías, mejorando el cuidado, que suministran los profesionales, desde sus diferentes roles.

\section{Metodología}

El abordaje del estudio, se hizo desde la investigación proyectiva, es el tipo de investigación, cualitativa, que permite elaborar un diagnóstico preciso de necesidades, para fundamentar una propuesta, un plan, un modelo, que puede ayudar en una necesidad de tipo práctico (Hurtado, 2007).

\section{Diseño}

El presente estudio se inscribe en el paradigma cualitativo porque trata de interpretar fenómenos que se presentan en las prácticas del cuidador de Enfermería es un diseño flexible y abierto, el desarrollo acciones es orientado por los participantes de la investigación con respecto a los eventos presentados. 


\section{Participantes}

Fueron los docentes y estudiantes de Enfermería, de una Institución de Educación Superior. La muestra, fue escogida por conveniencia, "sencillamente son casos disponibles a los cuales se tiene acceso" (Hernández et al., 2014, p. 571). En ese sentido, la muestra se configuró con 25 estudiantes de sexto semestre de Enfermería, de ambos sexos, cursando la asignatura de Seminario de Investigación; lo cual, fue indispensable para la selección del grupo, por estar estudiando metodología y construyendo su proyecto de grado; este es el criterio de inclusión, asumido para participar en el estudio.

\section{Técnicas e instrumentos}

Fueron la entrevista y el grupo focal, utilizando una guía de entrevista, estructurada con preguntas abiertas, como si fuera una conversación, con la finalidad de alcanzar información del participante en plena libertad, para expresar sus "conocimientos, pensamientos, sentimientos y emociones, mediante sus propias palabras" (Bisguerra 2004, p. 340).

La guía de grupo focal, se planeó como reuniones de grupo, donde los participantes intercambiaron experiencias, ideas, opiniones, críticas, compartieron otras visiones de la realidad de la investigación en enfermería, esta técnica proporciona conocimientos en cuanto a creencias y actitudes que subyacen en el comportamiento. (Rubím et al., 2013).

La validez y confiabilidad, hace referencia al nivel de credibilidad o veracidad de las descripciones, interpretaciones y conclusiones de un estudio. En esta investigación la confiabilidad, se verificó con la información tomada tal cual, como lo expresaron los participantes. Strauss y Corbin (2012), los resultados se relacionan, con la realidad de la investigación formativa es decir, motivar y desarrollar investigación cualitativa desde las prácticas formativas en el programa de Enfermería.

Para el análisis de la información obtenida, lo primero fue la organización de los datos, que consistió en transcribir a mano, al pie de la letra, los audios de las grabaciones, producto de las entrevistas y sesión de grupo focal; se realizó en orden cronológico, en el proceso de análisis de los datos, se buscó las ideas principales, valorando las 
expresiones, opiniones y necesidades sentidas por los educandos y educadores; el lenguaje utilizado por los informantes, fue claro y explícito, guiando el procedimiento de análisis, identificando conceptos y elementos importantes en los datos; en pocas palabras, la secuencia de interacciones, ideas centrales, surgieron como códigos, los cuales se estructuraron como categorías orientadoras de la investigación.

En los aspectos éticos, se garantizaron los principios de confidencialidad, ausencia de engaño; respecto a los participantes y a la información recibida, explicación sobre los resultados, solo se utilizarían con fines pedagógicos y académicos.

\section{Resultados}

Los resultados se presentan de acuerdo con el objetivo y pregunta que guiaron la investigación, en dirección de proponer la Sistematización de Experiencias, como una forma de hacer investigación cualitativa e indagar los conocimientos y práctica de la investigación cualitativa en estudiantes de enfermería.

Los participantes en el estudio, exteriorizaron desconocimiento y desmotivación en la práctica y utilización del enfoque cualitativo; debido a los mitos y creencias sobre esta metodología que producen desinterés en los estudiantes y docentes, se dice que la investigación cualitativa es un proceso: difícil, largo y dispendioso, esta información es trasmitida por los mismos estudiantes y docentes como una bola de nieve, poco a poco desde el primer semestre hasta el último.

Respecto a los conocimientos y prácticas de la Sistematización de Experiencias, como medio para producir conocimientos en Enfermería: los participantes, manifestaron, desconocer totalmente el tema, nunca han utilizado esta estrategia en producción de saberes; se observa, que estudiantes y docentes participantes en la investigación, tienen mayor motivación y prefieren la investigación cuantitativa, siendo que Enfermería es una disciplina que tiene un cuerpo de conocimientos, relacionado al cuidado de la salud de los seres humanos y el ambiente; existe la necesidad de aplicar ambos enfoques de investigación, para optimizar la práctica del cuidado. 
Finalmente, se expresa que en el programa no existe motivación para realizar estudios cualitativos, en los participantes se nota poco interés y lo expresaron diciendo que no encontraban quien les asesorara los proyectos de investigación bajo este paradigma. Asimismo, cuando se abordó el tema de sistematización de experiencias los estudiantes lanzaron expresiones como: "ni idea"“eso con que se come". Existe un desconocimiento total sobre este método que desarrolla el pensamiento y produce nuevos conocimientos originados de la práctica, como un camino de mejorar el cuidado de enfermería.

\section{Propuesta pedagógica}

Para organizar la conceptualización de sistematización de experiencias en la práctica de Enfermería se considera los siguientes componentes o ejes temáticos en la propuesta pedagógica: historia de la sistematización de experiencia en América Latina, definiciones, supuestos básicos, enfoques de sistematización de experiencias, proceso metodológico, puesta en marcha de un proyecto de sistematización de un caso; organización metodológica de fenómenos o eventos que se presentan en salud, descripción del fenómeno, referencias, confrontar la práctica con la teoría, conocimientos emanados de las experiencia; practicar aplicación de técnicas cualitativas, guías que faciliten la reconstrucción de prácticas de Enfermería, analizar situaciones problemas, narrativas, análisis documental; en resumen realizar estrategias pedagógicas y didácticas como: seminario, visitas a instituciones, domiciliarias, taller educativo, el método de caso, observación, participante, relación de ayuda todas aquellas prácticas que permiten entrar al enfoque de investigación cualitativa.

También es de suma importancia motivar a los estudiantes explicándoles el paso a paso de la metodología ayudándolos a atreverse a superar limitaciones asistiendolos frecuentemente en la producción del texto y las consultas en bases de datos.Ver tabla1. 


\section{Tabla 1}

Propuesta pedagógica sobre sistematización de experiencia

\section{ETAPAS DE LA PROPUESTA DE SISTEMATIZACIÓN DE EXPERIENCIAS}

\begin{tabular}{|c|c|}
\hline & Reflexiva \\
\hline Primera etapa & $\begin{array}{l}\text { Se reflexiona sobre la experiencia vivida, reconstrucción y } \\
\text { direccionamiento mediante las categorías que orientan la } \\
\text { sistematización. } \\
\text { Registrar de manera ordenada la experiencia que se va a } \\
\text { sistematizar. } \\
\text { Exponer la problemática existente. } \\
\text { Construcción de objetivos e interrogantes. } \\
\text { Describir la razón por las que elige la experiencia. } \\
\text { Fundamentación conceptual. } \\
\text { Dimensión ética } \\
\text { Recursos }\end{array}$ \\
\hline & Trabajo de campo \\
\hline
\end{tabular}

Segunda Etapa

Recogida de datos: identificar las técnicas los instrumentos y guías que faciliten la reconstrucción de la experiencia.

Seleccionar la información necesaria como condición sustancial.

\section{Analítica}

Tercera Etapa

Reordenamiento de la información de acuerdo con los objetivos del estudio.

Análisis de las categorías que orientan la sistematización.

Interpretación de la información

\section{Pedagógica}

Cuarta Etapa

Informe de la sistematización.

Conclusiones.

Aprendizajes.

Fuente: elaboración propia (2019). 
- Primera etapa: es reflexiva ayuda a dar claridad en los aspectos y elementos que muestra la práctica, es el punto de partida se definen los objetivos, se describe reflexivamente la experiencia que se va a retomar o reconstruir, con las bases conceptuales utilizando un proceso que permita presentar ordenadamente la situación problema con sus particularidades y características personales y el espacio donde se desarrolla la experiencia, se justifican las razones por las cuales se sistematiza determinada práctica, es necesario planear los recursos humanos institucionales y materiales para realizar el estudio. En esta primera parte es necesario precisar el componente ético consentimiento informado y otros elementos que tienen que ver con la confidencialidad de los datos encontrados; en este punto también se incluyen diferentes vicisitudes que influyen o que hayan ocurrido en la localidad.

- Segunda etapa: es la ubicación de la práctica que se desea sistematizar, teniendo presente diferentes elementos como la identificación de las categorías y subcategorías que orientan el estudio y los factores que influyen en la presentación de la situación problema, como afectan, las ideas, estrategias que se aplicaran en el estudio. Es necesario planificar las técnicas e instrumentos que permitirán recolectar la información necesaria, se escogen las personas afectadas con sus características socioeconómicas y culturales, sus interacciones y la forma como el ambiente interviene en su salud o enfermedad.

- Tercera etapa: se realiza con base en los aspectos anteriores se cumple un reordenamiento de la información a la luz de las categorías, el propósito y los interrogantes; efectuando un análisis de todas las condiciones que dan lugar al estudio, se aportan los elementos para formalizar la comprensión donde se revelan las interrelaciones de los componentes del medio y las personas.

La interpretación se hace buscando la correspondencia con las categorías y la parte teórica definida en el diseño, además se hace la comprensión de la experiencia con los elementos y conocimientos de una acción que está constituida por experiencias internas y externas resultantes de las interacciones personales. 
- Cuarta etapa: comprende la intervención pedagógica que consiste en construir una información detallada y organizada de todo el proceso, realizando retroalimentación y ajustes necesarios articulando los componentes, para obtener la validez y confiabilidad en coherencia con la información, se realiza el informe de la sistematización de experiencia describiendo claramente los resultados en forma ordenada verificando con otras experiencias para enriquecer la práctica. El documento construido debe elaborarse en forma creativa procurando expresar de forma clara los nuevos aprendizajes.

Ciertamente, la investigación formativa es la estrategia utilizada en las instituciones educativas y programas de Enfermería, para promover: actitudes, valores, métodos y técnicas que despiertan el espíritu de curiosidad y deseo de búsqueda permanente de conocimientos, favoreciendo el desarrollo de competencias cuando la incorpora en la atención de Enfermería.

\section{Discusión}

En el programa existe desconocimiento del enfoque cualitativo y de la sistematización de experiencias en la práctica de Enfermería, esto significa que se está desaprovechando esta forma de conocer y hacer en la práctica del cuidado, este enfoque permite la comprensión y entendimiento de situaciones, motivos y creencias, que están detrás de las acciones del ser humano; la sistematización de experiencias es valiosa en la formación de las personas, ayuda al desarrollo de la ciencia de Enfermera, produce conocimientos y teorías, para mejorar el cuidado, que suministran los profesionales en sus diferentes roles. (Gennaro y Fehder, 2011).

Así, la investigación cualitativa permite una reflexión permanente en los espacios de formación de los estudiantes, en la medida que se reflexiona las prácticas y procedimientos, en la relación de ayudar y la recuperación de los pacientes; lograr ser constructores de saberes y producir conocimientos nuevos mediante su propia práctica; recurriendo a la sistematización de experiencias como una estrategia pedagógica en enfermería para interpretar los fenómenosde la salud y enfermedad. 
La desmotivación existente en docentes y estudiantes en la práctica del enfoque cualitativo es un impedimento para el desarrollo de: actitudes, valores, métodos y técnicas que despiertan el espíritu de indagación y deseo de construir nuevos conocimientos para que el estudiante lo incorpore en la atención de Enfermería. La OPS y OMS. (2013), explican, que para bridar una atención humanizada, se requieren los distintos enfoques metodológicos, tanto en la academia como en la asistencia, para el abordaje de la problemática existente.

Es importante valorar la investigación cualitativa, tanto como la cuantitativa, los dos paradigmas, producen conocimientos en Enfermería, para comprender las necesidades de las personas y aprender el mejor modo de cuidar la vida y la enfermedad. El dilema entre ambos enfoques, no se resuelve en el debate teórico, sino en la realización de proyectos de investigación, que muestran la necesidad de complementar ambos enfoques en la búsqueda de comprender realidades concretas y complejas como es la problemática de la salud en Colombia y el mundo.

El desarrollo del conocimiento en Enfermería, requiere comprensión de la persona y sus experiencias individuales y colectivas las cuales no se pueden cuantificar, sino entrar en lo subjetivo mostrando entendimiento del sufrimiento, temores, dolores, creencias, desencuentros y mucha consideración sobre todo en los momentos de perdida de la salud física y mental.

De esta forma, la sistematización de experiencias, genera nuevas teorías producto de mucho trabajo, la CIE plantea la práctica basada en la investigación, para el desarrollo de la disciplina Enfermería por el avance que puede aportar la ciencia en el ejercicio de la profesión y los servicios de salud, contribuyendo con evidencia científica en la formación, la práctica y la gestión del cuidado en la promoción de la salud, prevención de la enfermedad y brindar una atención holística a las personas, familia, comunidad y ambiente.

El estudio permitió proponer una estrategia pedagógica, basada en la sistematización de experiencias, como una manera de hacer investigación cualitativa, en la promoción, prevención y atención de los seres humanos; es una didáctica en función del aprendizaje, 
en la medida que posibilita la formación de competencias. Está compuesta por cuatro etapas, es un proceso de reconstrucción reflexiva para innovar la práctica de Enfermería, mejorar la atención con estrategias participativas en salud para la formación de personas con capacidades para comprender e incidir en los diferentes campos de enfermería.

\section{Conclusiones}

La investigación formativa, es la estrategia utilizada en las instituciones educativas y programas de Enfermería, para promover: actitudes, valores, métodos y técnicas que despiertan el espíritu de curiosidad y deseo de búsqueda permanente de conocimientos, favoreciendo al estudiante y docente cuando es incorporado en la atención de la vida y la salud. En el programa de enfermería existe desconocimiento de la metodología cualitativa, lo que significa que es necesario buscar herramientas para promover este enfoque como una estrategia curricular, que permita a los estudiantes adquirir los conocimientos teóricos, conceptuales y las competencias para realizar estudios cualitativos.

Asimismo, existe desmotivación en los estudiantes y docentes hacia la práctica del enfoque cualitativo, generado por mitos e información errada trasmitida por los mismos estudiantes y docentes desde que inician el primer semestre hasta cuando llegan al trabajo de grado. Es necesario promover e iniciar trabajos de sensibilización y orientación del enfoque cualitativo con técnicas que despiertan el espíritu de curiosidad y deseo de búsqueda permanente de conocimientos, que favorezca al estudiante y paciente cuando la incorpore en la atención de Enfermería.

El estudio permitió proponer estrategias pedagógicas, basadas en la sistematización de experiencias, como una manera de hacer investigación cualitativa, para la promoción, de la salud, prevención de la enfermedad y la atención de los seres humanos. Esta didáctica se presenta en función del aprendizaje en la medida que posibilita la formación de competencias procedimentales, actitudinales y cognitivas.

Para terminar la propuesta, contiene cuatro etapas como un proceso de reconstrucción reflexiva y crítica en el quehacer de Enfermería para mejorar la práctica del cuidado, es una pedagogía nueva y participativa en salud, para la formación de sujetos críticos 
reflexivos y propositivos, con capacidades de comprender e incidir en los diferentes campos de Enfermería.

\section{Referencias bibliográficas}

Alarcón, M. y Astudillo, P. (2007). La Investigación en Enfermería. En revistas latinoamericana. Cienc. Enferm. Vol. 13 N.2 Supl.2. .Recuperado el 12 noviembre de 2016. http://dx.doi.org/10.4067/S071795532007000200004.

Bizquerra, R. (2004). Metodología de la investigación educativa. Primera edición. Editorial la Muralla.

Cifuentes, M. (2006). La sistematización de las prácticas en trabajo social: Una visión desde los proyectos sociales. Primera edición Manizales: Universidad de Caldas.

Galeano, M. (2013). Diseño de proyectos de investigación cualitativa. Octava reimpresión. Editorial Fondo Editorial Universidad Eafit.

Gennaro, S. y Fehder, W. (2011). Mejoramiento de la calidad de la difusión de la ciencia de la Enfermería. Editorial Universidad de la Sabana. RevistaAquichan; Vol.11-N3

Hurtado, J. (2007). El proyecto de investigación. Comprensión holística de la metodología de la investigación. Sexta edición. Ediciones Quirón.

Hernández, R., Fernández, C., Baptista, P. (2014). Metodología de la investigación. Cuarta edición. Printed by compañía editorial Ultra. D.F.

Mejía, M. (2011). La sistematización empodera y produce saber y conocimiento. Primera Edición. Desde Abajo.

OPS. OMS. (2013). Investigación cualitativa en enfermería metodología y didáctica. 
Rubim, E., Porto, J., Zárate, R. y González, L. (2013). Grupo focal en investigación cualitativa de Enfermería. Cap. 15. En Do Prado, M., De Souza, M., Monticelli, M. y Gómez, P. (Eds.), Investigación cualitativa en Enfermería. Metodología y didáctica. (Serie PALTEX Salud y Sociedad 2000, $\mathrm{N}^{\circ} 10$ p.187) ISBN 978-92-75-31817-1

Strauss, A. y Corbin, J. (2012). Bases de la investigación cualitativa. Técnicas y procedimientos para desarrollar la teoría fundamentada. Primera Edición. Editorial Universidad de Antioquia.

Triviño, Z. y Sanhueza, O. (2005). Paradigmas de investigación en Enfermería. Ciencia y Enfermería XI (1): 17-24, 2005. I.S.S.N. 0717 - 2079. https://www. redalyc.org/pdf/3704/370441790003.pdf

Vélez, C., Pico, M., Ariza,C., Castellano, F. y Salazar, A. (2014). Tendencias y Agenda de prioridades de investigación en unidades académicas de educación superior de Enfermería en Colombia. Cienc. Enf. Vol. 20. Recuperado el 5 noviembre de 2016.http://dx.doi.org/10.4067/S0717-95532014000300002. 